\title{
IVF in Developing Economies and Low Resource Countries: An Overview
}

\author{
Allahbadia G. N. \\ Published online: 28 September 2013 \\ (C) Federation of Obstetric \& Gynecological Societies of India 2013
}

Despite a record of 5 million IVF babies born in the world, the treatment of infertility by effective methods remains largely the preserve of developed countries [1]. Most infections causing tubal damage are preventable, and assisted conception can treat the infertility. However, assisted conception, despite being available for nearly three decades, is either unavailable or inaccessible to most residents of resource-poor countries. Provision of assisted reproductive technology (ART) to overcome both female and male infertility is in line with the reproductive rights agenda developed at the International Conference on Population and Development (ICPD) at Cairo 15 years ago [2]. In addition to the right to control fertility, reproductive rights must encompass the right to facilitate fertility when fertility is threatened. Facilitation of fertility may require resort to ART among both men and women. There is an increased need for low-cost procedures in treating infertility particularly in developing countries. One of the United Nation's Millennium Development Goals was for universal access to reproductive health care by 2015 , and WHO has recommended that infertility be considered a global health problem and stated the need for adaptation of ART in low-resource countries.

Allahbadia G. N. ( $₫)$, Medical Director

Rotunda-The Center for Human Reproduction, 36 Turner

Road, \#101, 1st Floor, B Wing, Bandra (W), Mumbai 400 050, India

e-mail: gautam@rotundaivf.com
Murage et al. [3] conducted a survey in a developing country (Kenya) to gage the extent of subfertility and the current state of ART service provision and explore factors limiting access to ART services. A total of 47 responses ( $25 \%)$ were received after completion of the survey. The overall rate of sub-fertility was $26.1 \%$ among the gynecology consultations, with $50 \%$ being attributed to tubal factors and $15 \%$ to male factors. Assisted reproductive service provision (IVF/intracytoplasmic sperm injection) was severely limited to only three units, despite the reported high rate of tubal disease [3]. The high cost of treatment, patients' limited finances, and limited local services were almost universally cited as the main barriers to ART services in Kenya. The authors summarized that the demand for ART in developing countries is never in doubt. Simplified, less costly, and more accessible ART approaches need to be considered in developing countries, even though the benefits and outcomes of such approaches may not be apparent immediately [3].

\section{Discussion}

Three ethical concerns are often mentioned specifically with regard to developing countries: (a) the "overpopulation argument"; (b) the limited resources argument; and (c) the ethical problem of poorly trained practitioners offering their services to unsuspecting and uninformed infertile individuals or couples. Each argument was explored in some detail in a landmark published study, with the conclusion that ethical 
problems do, in fact, exist but are not unique to developing countries alone [4]. Nevertheless, the difficulties relating to reproductive technologies are likely to be greater in the developing countries than in developed ones because of limited resources and a larger number of poor people residing in the former [4].

Intrauterine insemination was used long before the advent of in vitro fertilization. During the last 30 years, however, intrauterine insemination has evolved with the introduction of ovulation-stimulating protocols and sperm preparation methods taken from assisted reproduction techniques [5]. Costs have risen, but the success rate has not risen to the same extent. An African study group has developed a simple intrauterine insemination technique, which may be performed in developing countries, without the need of sophisticated equipment, costly materials, media, or disposable insemination catheters; it is quite inexpensive and may be performed by trained staff, such as nurses or midwives [6]. In this study, 20-27\% of the couples remained clinically pregnant after an average of 3.5-3.8 intrauterine inseminations procedures [6].

IVF is the definitive line of treatment for many couples. Stimulation cycles are associated with risks of ovarian hyperstimulation syndrome and multiple pregnancy. This study from Egypt evaluates the client acceptability of stimulated versus natural cycle IVF among couples attending one infertility clinic, with respect to cost and pregnancy outcome [7]. Of the patients who were indicated for IVF, $15 \%$ (16/ $107)$ canceled, due mostly $(12 / 16,75 \%)$ to financial reasons. The majority of patients who completed their IVF treatment $(82 / 91,90.1 \%)$ felt that the price of the medical service offered was high, and $68.1 \%$ (62/91) accepted the idea of having cheaper drugs with fewer side effects, but with possibly lower chances of pregnancy [7]. Natural cycle IVF has emerged as a potential alternative option that might be suitable for patients worldwide, especially in developing countries [7].

A Thai group presented their "Simplified IVF" program at Ramathibodi Hospital. Some steps of the conventional IVF procedures have been modified because of the limitation of resources. Simplification of procedures enabled the IVF service to be available in a center with limited resources without compromising the results [8]. Other advantages as outlined by the authors are improvement of the patient's convenience, cost savings, and less time consumed as well as being less stressful [8].

In India, we have been trying to make IVF more affordable to ensure a wider reach across the socio economic strata with introduction of cheaper and more patientfriendly Assisted Conception procedures without compromising on results [9]. Routine IVF (IVF), is slowly being challenged by simpler and more cost-effective methodologies. These include
- Natural cycle IVF (nIVF) [10].

- Minimal stimulation IVF (msIVF) [11, 12].

- IVF Lite (minimal stimulation IVF + vitrification + accumulation of embryos + remote embryo transfer) $(\mathrm{msIVF}+\mathrm{ACCUVIT}+\mathrm{rET})[9,13]$.

A minimal stimulation IVF cycle is defined either as

(a) a stimulation regimen in which gonadotropins are administered at a lower-than-usual dose and/or for a shorter duration throughout a cycle in which $\mathrm{GnRH}$ antagonist is given as co-treatment [14, 15]; or

(b) a stimulation in which oral compounds (e.g., antiestrogens) are used either alone or in combination with gonadotropins and GnRH-antagonists [16, 17].

Mild stimulation protocols reduce the mean number of days of stimulation, the total amount of gonadotropins used and the mean number of oocytes retrieved [11]. The proportion of high-quality and euploid embryos seems to be higher compared with conventional stimulation protocols, and the pregnancy rate per embryo transfer is comparable [10]. With the reduced costs, the better tolerability for patients, and the less time needed to complete an IVF cycle, these mild approaches are gaining a permanent foot-hold in cost-sensitive economies.

Intravaginal culture (IVC), also called INVO (intravaginal culture of oocytes), is an assisted reproduction procedure where oocyte fertilization and early embryo development are carried out within a gas permeable air-free plastic device, placed into the maternal vaginal cavity for incubation [18-21]. INVO can be performed in a physician's office or in a satellite facility of an IVF center. The INVO procedure consists of fertilization of oocyte(s) and early embryo development in the INVO cell device placed into the maternal vaginal cavity for incubation. The vaginal cavity replaces the complex in vitro fertilization (IVF) laboratory. Over 800 cycles have been published worldwide which showed a clinical pregnancy rate of $19.6 \%$ [18-21]. The participation of the patient in the process of fertilization and early embryo development is a psychological benefit that creates a high level of acceptance of INVO.

In a recent study the authors assessed the outcome of the INVO procedure, using the recently upgraded INVO cell device, in combination with a mild ovarian stimulation protocol [21]. A total of 125 cycles were performed. On average, 6.5 oocytes per cycle were retrieved, and a mean of 4.2 were placed per INVO cell device. The cleavage rate obtained after the INVO culture was $63 \%$. The procedure yielded 40, 31.2, and $24 \%$ of clinical pregnancy, live birth, and single live birth rates per cycle, respectively [21]. These published results hold promise that the INVO procedure is an effective alternative treatment option in assisted reproduction that shows comparable results to those reported for existing IVF techniques. 
A study performed last year in Belgium with another in vitro cheap culture device has shown that low-cost IVF for the developing and poor-resource countries is feasible and effective, with delivery rates not much different from those achieved in conventional IVF programs [22]. Klerkx et al. [10] led a 11-month prospective study at Genk in which oocytes were cultured according to (a) regular IVF culturing, or (b) the tWE lab IVF culture system (50/50 \%). The primary outcome parameter was embryo quality at day 3 ; secondary outcome parameters were embryo implantation rate and ongoing pregnancy rate. Only first IVF attempts in women $<36$ years with $\geq 8$ oocytes were included. Severe male factor infertility cases were excluded. They always performed single embryo transfer (SET). In their system, an optimal culture environment was reproducibly obtained without the need for medical gases, complex incubation equipment, and expensive infrastructure. Similar rates of fertilization and cleavage were observed in both groups. In two cases, no fertilization occurred, all embryos were cryopreserved in 3 cycles because of an increased risk of ovarian hyperstimulation. SET was performed in the remaining 35 IVF cycles. In 23 out of 35 cycles $(65.7 \%)$, the top quality embryo selected by an independent embryologist originated from the tWE lab system. In this group, the implantation rate was $34.8 \%$ $(8 / 23)$ with an ongoing pregnancy rate of $30.4 \%(7 / 23)$, and one miscarriage at 8 weeks gestation. Up to December 31, 2012, three healthy tWE lab babies have been born vaginally. This proof-of-principle study [10], as called by the investigators, suggests that infertility care may now be "universally accessible." The authors showed that the IVF methodology can be significantly simplified and can result in successful outcomes at levels that compare favorably to those obtained in high-resource programs. They concluded that the cost of their simplified culture system is between 10 and $15 \%$ of the current costs in Western-style IVF programs and computed that a cycle of IVF with the simplified procedure can be performed for around Rs 16,000 (Euros 200) [22].

The above study [22] was part of the Walking Egg Project, http://www.thewalkingegg.com/thewalkingegg, an international project aiming to raise awareness surrounding childlessness in resource-poor countries and to make infertility care, including ARTs, available and accessible for a much larger proportion of the world population.

The low-cost culture system developed by this USBelgian team of researchers, which can fit into a shirt pocket, is designed to operate anywhere, including off the grid, allowing it to be independent of the complex and costly infrastructure required by IFV programs in the developed world. The system uses low-cost components, does not require complex microprocessor-controlled incubators, and is a closed system that generates its own unique atmospheric and culture conditions required for normal fertilization and embryogenesis using inexpensive, common chemicals. The low-cost culture system is based on an incubator system consisting of two sealed glass tubes. A chemical reaction initiated by combining baking soda and citric acid in the first sealed glass tube generates an atmosphere that includes a specific percentage of carbon dioxide. The atmosphere is then transferred into the second glass tube holding the culture medium. The connection between the two glass tubes-needles and tubing-can easily be removed once the equilibrium between the two glass tubes is achieved. Oocytes and sperm are then injected by syringe into the tube containing the culture medium without disturbing the air environment inside the tube.

\section{Conclusion}

Worldwide, more than 80 million couples suffer from infertility, the majority being citizens of the developing economies. In the developing societies, childlessness is often highly stigmatized and leads to profound social suffering for infertile women in particular, yet most infertile people in the developing world have virtually no access to any effective treatment. Bilateral tubal blocks due to sexually transmitted diseases and pregnancy-related infections is the most common cause of infertility in the developing countries, a condition that is potentially treatable with ARTs. New reproductive technologies are either unavailable or very costly in the developing countries due to private monopoly [23]. Guidelines in the successful implementation of infertility care in low-resource areas include simplification of ART procedures like adopting IVF Lite [9], minimizing the complication rate of procedures like elimination of OHSS [7-9], providing training courses for health care workers and incorporating infertility treatment into sexual and reproductive health care programs [24]. One of the United Nation's Millennium Development Goals was for universal access to reproductive health care by 2015 , and WHO has recommended that infertility be considered a global health problem, and stated the need for adaptation of assisted reproduction technology in low-resource countries [25].

\section{References}

1. Sharma S, Mittal S, Aggarwal P. Management of infertility in low resource countries. BJOG. 2009;116(Suppl 1):77-83. doi: 10.1111/j.1471-0528.2009.02311.x.

2. Inhorn MC. Right to assisted reproductive technology: overcoming infertility in low-resource countries. Int $\mathrm{J}$ Gynaecol Obstet. 2009;106(2):172-4. doi:10.1016/j.ijgo.2009.03.034. 
3. Murage A, Muteshi MC, Githae F. Assisted reproduction services provision in a developing country: time to act? Fertil Steril. 2011;96(4):966-8. doi:10.1016/j.fertnstert.2011.07.1109.

4. Macklin RB. Reproductive technologies in developing countries. Bioethics. 1995;9(3-4):276-82.

5. Allahbadia G. Does the addition of gonadotropin-releasing hormone analogs improve the pregnancy rates in intrauterine insemination? J Obstet Gynaecol India. 2011;61(3):261-4.

6. Mve RN, Formigli L. Low cost, simple, intrauterine insemination procedure with unwashed centrifuged husband's sperm for developing countries. Afr J Reprod Health. 2012;16(4):175-9.

7. Shahin AY. The problem of IVF cost in developing countries: has natural cycle IVF a place? Reprod Biomed Online. 2007;15(1):51-6.

8. Rojanasakul A, Choktanasiri W, Suchartwatanachai C, et al. "Simplified IVF": program for developing countries. J Med Assoc Thai. 1994;77(1):12-8.

9. Allahbadia GN. IVF Lite: is this the future of assisted reproduction? J Obstet Gynaecol India. 2013;63(1):1-4. doi:10.1007/s13224013-0402-6.

10. Matsuura T, Takehara Y, Kaijima H, et al. Natural IVF cycles may be desirable for women with repeated failures by stimulated IVF cycles. J Assist Reprod Genet. 2008;25(4):163-7.

11. Kim CH, Kim SR, Cheon YP, et al. Minimal stimulation using gonadotropin-releasing hormone $(\mathrm{GnRH})$ antagonist and recombinant human follicle-stimulating hormone versus GnRH antagonist multiple-dose protocol in low responders undergoing in vitro fertilization/intracytoplasmic sperm injection. Fertil Steril. 2009;92(6):2082-4.

12. Teramoto S, Kato O. Minimal ovarian stimulation with clomiphene citrate: a large-scale retrospective study. Reprod Biomed Online. 2007;15(2):134-48.

13. Zhang J, Chang L, Sone Y, et al. Minimal ovarian stimulation (mini-IVF) for IVF utilizing vitrification and cryopreserved embryo transfer. Reprod Biomed Online. 2010;21(4):485-95.

14. Pelinck MJ, Vogel NE, Hoek A, et al. Cumulative pregnancy rates after three cycles of minimal stimulation IVF and results according to subfertility diagnosis: a multicentre cohort study. Hum Reprod. 2006;21(9):2375-83.
15. Weghofer A, Margreiter M, Bassim S, et al. Minimal stimulation using recombinant follicle stimulating hormone and a gonadotropin-releasing hormone antagonist in women of advanced age. Fertil Steril. 2004;81(4):1002-6.

16. Mohsen IA, El Din RE. Minimal stimulation protocol using Letrozole versus microdose flare up $\mathrm{GnRH}$ agonist protocol in women with poor ovarian response undergoing ICSI. Gynecol Endocrinol. 2013;29(2):105-8.

17. Williams SC, Gibbons WE, Muasher SJ, et al. Minimal ovarian hyperstimulation for in vitro fertilization using sequential clomiphene citrate and gonadotropin with or without the addition of a gonadotropin-releasing hormone antagonist. Fertil Steril. 2002;78(5):1068-72.

18. Bonaventura L, Ahlering P, Morris R, et al. The INVO cell, a new medical device for intra vaginal fertilization and culture. Fertil Steril. 2006;86(3):S164 supplement 2.

19. Frydman R, Ranoux C. INVO: a simple, low cost effective assisted reproductive technology. ESHRE Monogr. 2008;1:85-9.

20. Lucena E, Saa AM, Navarro DE, et al. The new era in ART: INVO. Fertil Steril. 2010;94(Suppl 4):S36.

21. Lucena E, Saa AM, Navarro DE, et al. INVO procedure: minimally invasive IVF as an alternative treatment option for infertile couples. Sci World J. 2012;2012:571596. doi:10.1100/2012/571596.

22. Klerkx EPF, Janssen M, van Blerkom J et al. O-014 First pregnancies with a simplified IVF procedure: a crucial step to universal and accessible infertility care Hum Reprod. Abstracts of the 29th Annual Meeting of the European Society of Human Reproduction and Embryology 2013; 28 suppl 1:i4-i6.

23. Nachtigall RD. International disparities in access to infertility services. Fertil Steril. 2006;85(4):871-5.

24. ESHRE Task Force on Ethics and Law, Pennings G, de Wert G, et al. Providing infertility treatment in resource-poor countries. Hum Reprod. 2009;24(5):1008-11. doi:10.1093/humrep/den503.

25. Hammarberg K, Kirkman M. Infertility in resource-constrained settings: moving towards amelioration. Reprod Biomed Online. 2013;26(2):189-95. doi:10.1016/j.rbmo.2012.11.009. 